\title{
Discrete Degenerate Representations of Non-Compact Unitary Groups
}

\author{
R. RĄCZKA* and J. FISCHER** \\ International Centre for Theoretical Physics, Trieste
}

Received April 15, 1966

\begin{abstract}
Two degenerate principal series of irreducible unitary representations of an arbitrary non-compact unitary group $U(p, q)$ are derived. These series are determined by the eigenvalues of the first and second-order invariant operators, which are shown to possess a discrete spectrum. The explicit form of the corresponding harmonic functions is derived and the properties of the discrete representations are discussed in detail. Moreover, in the Appendix, we derive the properties of the corresponding degenerate representations of an arbitrary compact $U(p)$ group.
\end{abstract}

\section{Introduction}

Irreducible unitary representations of semi-simple Lie groups can be constructed in the Hilbert space $\mathscr{H}\left(X_{i}\right)$ of the functions defined in a domain $X_{i}$, which is some homogeneous space of the type

$$
X_{i}=G / G_{i},
$$

$G_{i}$ being a closed subgroup of $G$. In the case of the non-compact unimodular unitary groups $S U(p, q)$ we can construct two classes of homogeneous spaces having a compact and non-compact stability group, respectively. The first class contains the following symmetric Cartan spaces (see [1], Chap. IX):

Table

\begin{tabular}{lll}
\hline$X$ & rank & dimension of $X$ \\
\hline$S U(n) / S O(n)$ & $n-1$ & $\frac{1}{2}(n-1)(n+2)$ \\
$S U(2 n) / S p(n)$ & $n-1$ & $(n-1)(2 n+1)$ \\
$S U(p+q) / S[U(p) \times U(q)]$ & $\min (p, q)$ & $2 p q$ \\
$S U(p, q) / S[U(p) \times U(q)]$ & $\min (p, q)$ & $2 p q$
\end{tabular}

* On leave of absence from Institute of Nuclear Research, Warsaw, Poland.

** On leave of absence from Institute of Physics of the Czechoslovak Academy of Sciences, Prague, Czechoslovakia.

17 Commun. math. Phys., Vol. 3 
The spaces of the second class have been classified by Rosenferid [2] and FedYenko [3]. For our purposes the following spaces of this class are important:

$$
\begin{array}{ll}
X_{k, s}=S U(p, q) / S[U(k, s) \times U(p-k, q-s)] & p \geqq k \geqq 0 \\
& q \geqq s \geqq 0 .
\end{array}
$$

The key theorem, which relates the geometry of a homogeneous space $X$ to the properties of representations in the Hilbert space $\mathscr{H}(X)$, is the Gel'fand theorem [4]. It states that the number of invariant differential operators whose eigenvalues determine the irreducible unitary representations realized in $\mathscr{H}(X)$ is equal to the rank of the symmetric space $X$ (independently of the magnitude of the rank of the group $)^{1}$. If the rank of the space $X$ is one, then the ring of invariant operators in the enveloping algebra is generated by the so-called LaplaceBeltrami operator ${ }^{2}$

$$
\Delta(X)=\frac{1}{\sqrt{|\bar{g}|}} \partial_{\alpha} g^{\alpha \beta}(X) \sqrt{|\bar{g}|} \partial_{\beta}
$$

where $g_{\alpha \beta}(X)$ is the metric tensor on the space $X$ and $\bar{g}=\operatorname{det}\left\{g_{\alpha \beta}\right\}$. This operator is equal to the second-order Casimir operator $Q_{2}=g_{i k} Z^{i} Z^{k}$ $\left(Z_{i}\right.$ being the generators of $G$ ) of the group $G$ with the condition that the Riemannian metric tensor $g_{\alpha \beta}(X)$ on $X$ is induced by the Cartan metric tensor $g_{i k}$ in the Lie algebra $R$ of the group $G$ (see [1] Chap. X, $\S 7$ ).

In the present paper we consider the properties of the discrete degenerate representations of an arbitrary non-compact unitary group $U(p, q)$ which are determined by the eigenvalues of the first and secondorder invariant operators $\hat{M}$ and $\Delta(X)$, where

$$
\hat{M}=\sum_{i=1}^{p+q} Z_{i}^{c},
$$

$Z_{i}^{c}$ being the generators of a Cartan subgroup of $U(p, q)$. These representations are realized in the Hilbert spaces $\mathscr{H}\left(X_{ \pm}^{p_{ \pm}{ }^{b}}\right)$ of functions defined in the domains $X_{+}^{p, q}$ or $X_{\underline{p}}^{p, q}$,

$$
X_{+}^{p, q}=U(p, q) / U(p-1, q), \quad X \underline{p, q}=U(p, q) / U(p, q-1),
$$

the dimension of the spaces $X_{ \pm}^{p, q}$ being equal to $2(p+q)-1$. It has been shown by RosENFELD ([5], p. 621) that the group $U(p, q)$ acts transitively on these manifolds.

The full problem of the construction of the degenerate representations of an arbitrary $U(p, q)$ group is solved by the following steps:

(i) The construction of a convenient co-ordinate system on $X$, in which the metric tensor $g_{\alpha \beta}(X)$ is diagonal.

1 The rank of a symmetric space $X$ is the number of independent invariants of any pair of points $x, y \in X$ with respect to the action of $G$ on $X$.

2 If no other indication is given we shall employ the Einstein summation convention. 
(ii) The solution of the eigenvalue problem for the invariant operators:

$$
\begin{aligned}
\Delta(X) \Psi_{M}^{\lambda} & =\lambda \Psi_{M}^{\lambda} \\
\hat{M} \Psi_{M}^{\lambda} & =M \Psi_{M}^{\lambda} .
\end{aligned}
$$

(iii) The proof of the irreducibility and unitarity of the representations related to the set of harmonic functions $\Psi_{M}^{\lambda}$.

The properties of the discrete series of degenerate representations of the non-compact $U(p, q)$ groups are rather different if $p \geqq q>1$ or if $p>q=1$ or $p=q=1$. In Section 2 we give the solution of the problem for $p \geqq q>1$, i.e., the explicit form of harmonic functions and the character of the spectra of $\lambda$ and $M$. The cases $p>q=1$ and $p=q=1$ are studied in the same manner in Section 3. In Section 4 we give the proof of irreducibility and unitarity of the representations so obtained, while in Section 5 we discuss some of their more general properties. Finally, the Appendix contains the derivation and discussion of the properties of the corresponding degenerate irreducible unitary representations of an arbitrary compact $U(p)$ group.

In a series of papers we shall give the solution of the following problems:

I. The construction of the discrete degenerate representations of the $U(p, q)$ groups;

II. The construction of the continuous degenerate representations of the $U(p, q)$ groups;

III. The derivation of the Gel'fand-Graev transforms [6] which are related to the irreducible unitary representations of the $U(p, q)$ groups;

IV. The decomposition of the direct product of two degenerate representations of the $U(p, q)$ group into irreducible components;

$V$. The construction of other series of degenerate irreducible representations of the $U(p, q)$ groups $^{3}$.

Finally, let us mention that in the present paper we talk about a representation of the $U(p, q)$ group in a Hilbert space $\mathscr{H}(X)$ although, strictly speaking, we deal with a local representation determined on a dense set in $\mathscr{H}(X)$. However, we shall show in Part III that from the local irreducibility and unitarity the global irreducibility and unitarity follow.

\section{Discrete degenerate representations of the $U(p, q)$ group $(p \geqq q>1)$}

The set of harmonic functions which creates a basis for the representations under consideration is given by the set of simultaneous solutions of

${ }^{3}$ In the present paper we consider the degenerate irreducible representations of $U(p, q)$ determined by two invariant numbers only. In Part V, we shall consider degenerate representations of $U(p, q)$ determined by more than two invariant numbers. 
the equations (1.6) and (1.7). To obtain an explicit solution of these equations it is necessary to introduce a convenient model of the spaces $X_{+}^{p, q}$ and $X^{p}, q$, i.e., a manifold which has the same dimension and the same stability group as $X_{ \pm}^{p, q}$ and on which the group $U(p, q)$ acts transitively.

The model of $X_{ \pm}^{p, q}$ can be realized by the hypersurface in the $(p+q)$ dimensional complex space $C^{p+q}$ which is determined by the equation

$$
z^{1} \bar{z}^{1}+z^{2} \bar{z}^{2}+\cdots+z^{p} \bar{z}^{p}-z^{p+1} \bar{z}^{p+1}-\cdots-z^{p+q} \bar{z}^{p+q}= \pm 1 .
$$

Besides the space $C^{p+q}$ we shall also consider the flat Minkowski space $M^{2 p, 2 q}$ defined by the relations

$$
\begin{aligned}
x^{2 k-1} & =\operatorname{Re} z^{k} \quad z^{k} \in C^{p+q} ; x^{2 k-1}, x^{2 k} \in M^{2 p, 2 q}, \\
x^{2 k} & =\operatorname{Im} z^{k} \quad k=1,2, \ldots p+q .
\end{aligned}
$$

The solution of the eigenvalue problem is considerably facilitated if a system of co-ordinates is chosen such that the metric tensor $g_{\alpha \beta}\left(X_{ \pm}^{p, q}\right)$ on the hypersurface $X_{ \pm}^{p, q}$ induced by the metric tensor $g_{a b}\left(M^{2 p, 2 q}\right)$ on $M^{2 p, 2 q}$ is diagonal. As it was shown in a previous paper [7], the so-called biharmonic co-ordinate system turns out to be especially convenient for this purpose. We shall construct it with the help of a recursion prescription: first we construct the coordinate system for the compact submanifold satisfying the equation

$$
z^{1} \bar{z}^{1}+\cdots+z^{p} \bar{z}^{p}=1
$$

which is a homogeneous manifold with respect to the action of the compact subgroup $U(p)$ of $U(p, q)$. If we suppose that we have constructed the coordinate system for $z^{\prime \prime 1}, \ldots z^{\prime \prime l}, l<p$, then the co-ordinate system for the variables $z^{\prime 1}, \ldots z^{\prime l+1}$ is given by

$$
\begin{aligned}
& z^{\prime i}=z^{\prime i} \sin \vartheta^{l+1} \quad 0 \leqq \varphi^{i} \leqq 2 \pi \quad i=1,2, \ldots l \\
& z^{\prime l+1}=e^{i \varphi^{l+1}} \cos \vartheta^{l+1} \quad 0 \leqq \vartheta^{j} \leqq \frac{\pi}{2}, \quad j=, 2, \ldots l .
\end{aligned}
$$

Therefore, putting $z^{1}=e^{i \varphi^{1}}$ for $p=1$ and applying successively the procedure (2.4) we obtain the co-ordinate system for the manifold determined by Eq. (2.3) for an arbitrary $p=1,2, \ldots$. We shall denote the corresponding set of angles by $\omega \equiv\left\{\varphi^{1}, \ldots \varphi^{p}, \vartheta^{2}, \ldots \vartheta^{p}\right\}$.

In the same way, the co-ordinate system for the variables $z^{p+1}, \ldots, z^{p+q}$ satisfying the equation

$$
z^{p+1} \bar{z}^{p+1}+\cdots+z^{p+q} \bar{z}^{p+q}=1
$$

is also constructed. The corresponding set of angles will be denoted by $\tilde{\omega} \equiv\left\{\tilde{\varphi}^{1}, \ldots, \tilde{\varphi}^{q}, \tilde{\vartheta}^{2}, \ldots \tilde{\vartheta}^{q}\right\}$. Finally, the complete co-ordinate system on $X_{+}^{p, q}$ is created by

$$
\begin{array}{ll}
z^{i}=z^{\prime} \operatorname{ch} \theta & i=1,2, \ldots p \\
z^{l}=z^{\prime} \operatorname{sh} \theta & l=p+1, \ldots p+q
\end{array} \quad 0 \leqq \theta<\infty .
$$


The metric tensor $g_{\alpha \beta}\left(X_{+}^{p, q}\right)$ on the space $X_{+}^{p, q}$ is given by

$g_{\alpha \beta}\left(X_{+}^{p, q}\right)=\sum_{k, l=1}^{2(p+q)} g_{k l}\left(M^{2 p, 2 q}\right) \partial_{\alpha} x^{k} \partial_{\beta} x^{l} \quad \alpha, \beta=1,2, \ldots 2(p+q)-1$.

where $\partial_{\alpha}, \alpha=1,2, \ldots 2(p+q)-1$ denotes partial differentiation with respect to the angles $\varphi^{1}, \varphi^{2}, \ldots, \varphi^{p}, \tilde{\varphi}^{1}, \ldots, \tilde{\varphi}^{q}, \vartheta^{2}, \ldots, \vartheta^{p}, \tilde{\vartheta}^{2}, \ldots, \tilde{\vartheta}^{q}, \theta$.

Consider first the equations (1.6) and (1.7) on the homogeneous space $X_{+}^{p, q}$. Using formulae (1.3) and (1.4) we find that the invariant operators $\Delta\left(X_{+}^{p, q}\right)$ and $\hat{M}\left(X_{+}^{p, q}\right)$ can be expressed in terms of the biharmonic coordinates in the following way

$$
\begin{aligned}
\Delta\left(X_{+}^{p, q}\right)= & -\frac{1}{\operatorname{ch}^{2 p-1} \theta \operatorname{sh}^{2 q-1} \theta} \frac{\partial}{\partial \theta} \operatorname{ch}^{2 p-1} \theta \operatorname{sh}^{2 q-1} \theta \frac{\partial}{\partial \theta}+ \\
& +\frac{\Delta\left(X^{p}\right)}{\operatorname{ch}^{2} \theta}-\frac{\widetilde{\Delta}\left(X^{q}\right)}{\operatorname{sh}^{2} \theta} \\
\hat{M}_{p+q} & =\hat{M}_{p}+\hat{\bar{M}}_{q}, \quad \hat{M}_{p}=-i \sum_{k=1}^{p} \frac{\partial}{\partial \varphi^{k}}, \quad \hat{\bar{M}}_{q}=-i \sum_{l=1}^{q} \frac{\partial}{\partial \widetilde{\varphi}^{l}}
\end{aligned}
$$

where $\Delta\left(X^{p}\right), \hat{M}_{p}$ and $\tilde{\Delta}\left(X^{q}\right), \hat{\tilde{M}}_{q}$ are the invariant operators of the compact unitary group $U(p)$ and $U(q)$ respectively and $X^{a} \equiv X^{a, 0}$. The eigenfunctions of the operators $\Delta\left(X^{p}\right)$ and $\hat{M}_{p}$ for an arbitrary $U(p)$ group are given explicitly in the Appendix.

If we represent the simultaneous eigenfunctions of the operators (2.8) and (2.9) as a product of eigenfunctions of $\Delta\left(X^{p}\right)$ and $\hat{M}_{p}$ times eigenfunctions of $\widetilde{\Delta}\left(X^{q}\right)$ and $\hat{\bar{M}}_{q}$ times an unknown function $\psi_{J_{p}}^{\lambda}, \tilde{J}_{q}(\theta)$, we obtain the following equation for $\psi_{J_{p}, \tilde{J}_{q}}^{\lambda}(\theta)$

$$
\begin{aligned}
& {\left[-\frac{1}{\operatorname{ch}^{2 p-1} \theta \operatorname{sh}^{2 q-1} \theta} \frac{d}{d \theta} \operatorname{ch}^{2 p-1} \theta \operatorname{sh}^{2 q-1} \theta \frac{d}{d \theta}-\right.} \\
& \left.-\frac{J_{p}\left(J_{p}+2 p-2\right)}{\operatorname{ch}^{2} \theta}+\frac{\tilde{J}_{q}\left(\tilde{J}_{q}+2 q-2\right)}{\operatorname{sh}^{2} \theta}-\lambda\right] \psi_{J_{p}, \tilde{J}_{q}}^{\lambda}=0
\end{aligned}
$$

where $-J_{p}\left(J_{p}+2 p-2\right)$ and $-\tilde{J}_{q}\left(\tilde{J}_{q}+2 q-2\right)$ are eigenvalues of the operators $\Delta\left(X^{p}\right)$ and $\widetilde{\Delta}\left(X^{q}\right)$ respectively, with $J_{p}$ and $\tilde{J}_{q}$ being certain non-negative integers for $p, q>1$ (see Appendix).

The left-invariant Riemannian measure $d \mu\left(X_{+}^{p, q}\right)$ on the manifold $X_{+}^{p, q}$ is given by

$d \mu\left(X_{+}^{p, q}\right)=\sqrt{\bar{g}\left(X_{+}^{p, q}\right)} d \omega d \tilde{\omega} d \theta$

$$
=d \mu\left(X^{p}\right) d \tilde{\mu}\left(X^{q}\right) \operatorname{ch}^{2 p-1} \theta \operatorname{sh}^{2 q-1} \theta d \theta
$$

where

$$
\begin{aligned}
& d \mu\left(X^{p}\right)=\prod_{\sigma=2}^{p} \sin ^{2 \sigma-3} \vartheta^{\sigma} \cos \vartheta^{\sigma} d \vartheta^{\sigma} \prod_{\varrho=1}^{p} d \varphi^{\varrho} \\
& d \tilde{\mu}\left(X^{q}\right)=\prod_{\sigma=2}^{q} \sin ^{2 \sigma-3} \tilde{\vartheta}^{\sigma} \cos \tilde{\vartheta}^{\sigma} d \tilde{\vartheta}^{\sigma} \prod_{\varrho=1}^{q} d \tilde{\varphi}^{\varrho}
\end{aligned}
$$


As the eigenfunctions of the compact operators $\Delta\left(X^{p}\right), \hat{M}_{p}$ and $\widetilde{\Delta}\left(X^{q}\right), \hat{\bar{M}}_{q}$ are square integrable with respect to the measure $d \mu\left(X^{p}\right)$ and $d \tilde{\mu}\left(X^{q}\right)$, respectively, the problem of finding discrete series of the degenerate representations reduces to that of finding such solutions of equation (2.10) which are square integrable with respect to the measure

$$
d \mu(\theta)=\operatorname{ch}^{2 p-1} \theta \operatorname{sh}^{2 q-1} \theta d \theta
$$

which is induced by the measure (2.11).

The solution of (2.10) which is regular at $\theta=0$ is given by

$$
\begin{aligned}
& \psi_{J_{p}, \tilde{J}_{q}}^{\lambda}(\theta)=\operatorname{th}^{\tilde{J}_{q}} \theta \operatorname{ch}^{-\alpha} \theta \times \\
& \times{ }_{2} F_{1}\left(\frac{\tilde{J}_{q}-J_{p}+\alpha}{2}-p+1, \frac{\tilde{J}_{q}+J_{p}+\alpha}{2} ; \tilde{J}_{q}+q ; \operatorname{th}^{2} \theta\right)
\end{aligned}
$$

where $\alpha=p+q-1+\sqrt{(p+q-1)^{2}-\lambda}>0$. We obtain a square integrable solution regular at $\theta \rightarrow \infty$ if we impose the following condition on the parameters of ${ }_{2} F_{1}$

$$
\frac{1}{2}\left(\tilde{J}_{q}-J_{p}+\alpha-2 p+2\right)=-n
$$

where $n$ is an arbitrary non-negative integer. From this condition we can find that the discrete spectrum $\lambda$ of the operator $\Delta\left(X_{+}^{p, q}\right)$ is of the form $\lambda=-L(L+2 p+2 q-2) \quad L=-(p+q)+2,-(p+q)+3, \ldots$

where

$$
L=J_{p}-\tilde{J}_{q}-2 q-2 n .
$$

Finally, the complete set of orthonormal square integrable functions related to definite values of invariant numbers $L$ and $M$ is given by the following formula

$\mathbf{Y}_{M, M_{1}, \ldots, M_{p}, \tilde{M}_{1}, \ldots, \tilde{M}_{q}}^{L, J_{2}, \ldots, J_{p}, \tilde{J}_{q}}(\Omega, \widetilde{\Omega}, \theta)=V_{J_{p}, \tilde{J}_{q}}^{L}(\theta) \mathbf{Y}_{M_{1}, \ldots M_{p}}^{J_{2}, \ldots J_{p}}(\Omega) \mathbf{Y}_{\tilde{M}_{1}, \ldots \tilde{M}_{q}}^{\tilde{J}_{2}, \ldots \tilde{J}_{q}}(\widetilde{\Omega})$

where $V_{J_{p}, \tilde{J}_{q}}^{L}(\theta)$ are solutions of equation (2.10) given by

$$
\begin{aligned}
& V_{J_{p}, \tilde{J}_{q}}^{L}(\theta)=\frac{1}{\sqrt{N}} \operatorname{th}^{\tilde{J_{q}}} \theta \mathrm{ch}^{-(L+2 p+2 q-2)} \theta \times \\
& \times{ }_{2} F_{1}\left(\frac{\tilde{\tilde{J}_{q}}-J_{p}+L}{2}+q, \frac{\tilde{J_{q}}+J_{p}+L}{2}+p+q-1 ; \tilde{J}_{q}+q ; \operatorname{th}^{2} \theta\right) \\
& N=\frac{\Gamma\left(\frac{1}{2}\left(J_{p}-\tilde{J}_{q}-L\right)-q+1\right) \Gamma^{2}\left(\tilde{J}_{q}+q\right) \Gamma\left(\frac{1}{2}\left(L-\tilde{J}_{q}+J_{p}\right)+p\right)}{2(L+p+q-1) \Gamma\left(\frac{1}{2}\left(J_{p}+\tilde{J}_{q}+L\right)+p+q-1\right) \Gamma\left(\frac{1}{2}\left(J_{p}+\tilde{J}_{q}-L\right)\right)}
\end{aligned}
$$

(see [8]). For definite $L$, condition (2.17) imposes now the following restriction on $J_{p}$ and $\tilde{J}_{\alpha}$

$$
J_{p}-\tilde{J}_{q}=L+2 q+2 n \quad n=0,1,2, \ldots .
$$


The functions

with

$$
\mathbf{Y}_{M_{1}, \ldots, M_{p}}^{J_{2}, \ldots, J_{p}}(\Omega)=\frac{1}{\sqrt{N_{p}}} \prod_{l=1}^{p} e^{i \mu_{l} \phi^{l}} \prod_{k=2}^{p} \sin ^{2-k} \vartheta^{k} \frac{1}{d_{\alpha_{k}, \beta_{k}}^{\frac{1}{2}\left(J_{k}+k-2\right)}}\left(2 \vartheta^{k}\right)
$$

$$
\begin{aligned}
\mu_{l}= & M_{l}-\frac{l}{p} M_{p} \quad l=1,2, \ldots, p-1 \quad \mu_{p}=\frac{1}{p} M_{p} \\
\alpha_{k}= & \frac{1}{2}\left(M_{k}-M_{k-1}+J_{k-1}+k-2\right) \quad k=2, \ldots p \\
\beta_{k}= & \frac{1}{2}\left(M_{k}-M_{k-1}-J_{k-1}-k+2\right) \quad J_{1} \equiv M_{1} \\
& N_{p}=(2 \pi)^{p} p \underset{k=2}{\prod}\left(J_{k}+k-1\right)^{-1}
\end{aligned}
$$

are simultaneous eigenfunctions of the invariant operators $\Delta\left(X^{p}\right), \widehat{M}_{p}$ and their form is derived in the Appendix. (The functions $\mathbf{Y}_{\tilde{M}_{1}, \ldots . \tilde{J_{q}}}^{{\tilde{J_{q}}}_{2}}(\widetilde{\Omega})$ are obtained, naturally, only by an appropriate change of the corresponding symbols.) The integers $M_{i}, i=1,2, \ldots p$ and the nonnegative integers $J_{k}, k=2,3, \ldots p$ are restricted by the following conditions :

$$
\begin{array}{cc}
\left|M_{2}-M_{1}\right|+\left|M_{1}\right|=J_{2}-2 n_{2} & n_{2}=0,1, \ldots, \frac{1}{2}\left(J_{2}-\left|M_{2}\right|\right) \\
\left|M_{3}-M_{2}\right|+J_{2}=J_{3}-2 n_{3} & n_{3}=0,1, \ldots, \frac{1}{2}\left(J_{3}-\left|M_{3}\right|\right) \\
\left|M_{4}-M_{3}\right|+J_{3}=J_{4}-2 n_{4} & n_{4}=0,1, \ldots, \frac{1}{2}\left(J_{4}-\left|M_{4}\right|\right) \\
\vdots & \\
\left|M_{p}-M_{p-1}\right|+J_{p-1}=J_{p}-2 n_{p} & n_{p}=0,1, \ldots, \frac{1}{2}\left(J_{p}-\left|M_{p}\right|\right) .
\end{array}
$$

Analogous relations among the integers $\tilde{M}_{i}, i=1,2, \ldots q$ and the nonnegative integers $\tilde{J}_{k}, k=2,3, \ldots q$ are obtained from $(2.23)$ by replacing $M_{i}, J_{k}, n_{k}$ and $p$ by $\tilde{M}_{i}, \tilde{J}_{k}, \tilde{n}_{k}$ and $q$ respectively.

As it is explained in the Appendix, it is sometimes convenient to pass from the sets $\omega, \tilde{\omega}$ of angles to new sets $\Omega, \widetilde{\Omega}$ defined by

where

$$
\begin{aligned}
\Omega & \equiv\left\{\phi^{1}, \phi^{2}, \ldots, \phi^{p}, \vartheta^{2}, \ldots, \vartheta^{p}\right\} \\
\Omega & \equiv\left\{\tilde{\phi}^{1}, \widetilde{\phi}^{2}, \ldots, \tilde{\phi}^{q}, \tilde{\vartheta}^{2}, \ldots, \tilde{\vartheta}^{q}\right\}
\end{aligned}
$$

$$
\begin{array}{ll}
\phi^{l}=\varphi^{l}-\varphi^{l+1} \quad l=1,2, \ldots p-1 \\
\phi^{p}=\varphi^{1}+\varphi^{2}+\cdots+\varphi^{p} \\
\tilde{\phi}^{j}=\tilde{\varphi}^{j}-\tilde{\varphi}^{j+1} \quad j=1,2, \ldots q-1 \\
\tilde{\phi}^{q}=\tilde{\varphi}^{1}+\tilde{\varphi}^{2}+\cdots+\tilde{\varphi}^{q} .
\end{array}
$$

The general solution (2.18), (2.21) has already been written in terms of these new variables. 
The closed linear envelope of functions (2.18) creates the Hilbert space $\mathscr{H}_{M}^{L}\left(X_{+}^{p, q}\right)$ of functions $f(X)$ with the scalar product determined by the measure (2.11):

$$
(\varphi, \chi)=\int \varphi(x) \bar{\chi}(x) d \mu\left(X_{+}^{p, q}\right) .
$$

The space $\mathscr{H}_{M}^{L}\left(X_{+}^{p, q}\right)$ creates the representation space for the group $U(p, q)$ because for any generator $Z_{\alpha}$ belonging to the Lie algebra of $U(p, q)$ and for any function $p \in \mathscr{H}_{\mathcal{M}}^{L_{\alpha}}\left(X_{+}^{p, q}\right)$ we have

$$
\left[\Delta, Z_{\alpha}\right] \varphi=0, \quad\left[\hat{M}, Z_{\alpha}\right] \varphi=0 .
$$

In Section 4 we prove that the representation related to the set of harmonic functions (2.18) is irreducible and unitary. We shall denote this series of representations by $D_{M}^{L}\left(X_{+}^{p, q}\right)$ or briefly by $D_{M}^{L}\left(X_{+}\right)$.

In a similar way, we obtain the corresponding series $D_{M}^{L}\left(X^{p, q}\right)$ of discrete degenerate representations related to the homogeneous space $X^{p, q}$ given by expression (1.3). The corresponding set of harmonic functions is obtained from (2.18) simply by replacing $V_{J_{p} \tilde{J}_{q}}^{L}(\theta)$ on the right-hand side of $(2.18)$ by $V_{\tilde{J}_{q} J_{p}}^{L}(\theta)$. Simultaneously the role of the numbers $J_{p}, p$ and $\tilde{J}_{q}, q$ is interchanged in the formulae (2.11), (2.13) to (2.17), (2.19) and (2.20). The representations $D_{M}^{L}\left(X_{+}^{p, q}\right)$ and $D_{M}^{L}\left(X^{p}, q\right)$ are non-equivalent, except for the case $p=q$, when both Hilbert spaces coincide.

The structure of the Hilbert spaces $\mathscr{H}_{M}^{L}\left(X_{ \pm}^{p, q}\right)$ is very simple. As it follows from the form of the harmonic functions (2.18), the whole Hilbert space $\mathscr{H}_{M}^{L}$ can be represented as a direct sum of the form

$$
\begin{aligned}
& \mathscr{H}_{M}^{L}\left(X_{+}\right)=\sum_{\substack{\tilde{J}_{q}=0 \\
\left(J_{p}-\tilde{J}_{q}-L \text { even }\right)}}^{\infty} \sum_{J_{p}=L+\tilde{J}_{q}+2 q}^{\infty} \sum_{\substack{M_{p}=-J_{p} \\
\left(M_{p}+J_{p} \text { even }\right)}}^{\sum_{\substack{\left.\tilde{M}_{q} \\
\tilde{M}_{q}=-\tilde{J}_{q} \text { even }\right)}}^{\sum^{J_{q}}}} \oplus \\
& \oplus \underset{M M_{p} \tilde{M}_{q}}{\stackrel{H_{p} \tilde{J}_{q}}{\tilde{I}_{q}}}\left(X_{+}\right) \delta_{M, M_{p}+\tilde{M}_{q}}
\end{aligned}
$$

where $\mathscr{H}_{M M_{p}}^{L J_{p} \tilde{J}_{q}} \tilde{M}_{q}\left(X_{+}\right)$is a subspace of $\mathscr{H}_{M}^{L}\left(X_{+}\right)$in which the irreducible unitary representation of the maximal compact subgroup $U(p) \times U(q)$ determined by the invariant numbers $J_{p}, M_{p}$ and $\tilde{J}_{q}, \tilde{M}_{q}$ acts. The formula (2.28) represents in fact the decomposition of the representation of the $U(p, q)$ group determined by $L$ and $M$ with respect to the representations of its maximal compact subgroup. A similar formula holds also for the decomposition of $\mathscr{H}_{M}^{L_{M}}\left(X_{-}\right)$.

The structure of the Hilbert spaces $\mathscr{H}_{M}^{L}\left(X_{ \pm}^{p, q}\right)$ can be represented with the help of a three-dimensional net with the co-ordinates $J_{p}, \tilde{J}_{q}$ and $M_{p}$, say $\left(\tilde{M}_{q}=M-M_{p}\right)$, in which every knot represents one subspace $\mathscr{H}_{M M_{p} \tilde{M}_{q}}^{L J_{p} \tilde{J}_{q}}\left(X_{ \pm}^{p, q}\right)$ of $\mathscr{H}_{M}^{L}\left(X_{ \pm}^{p, q}\right)$. To construct the net in the case of the 
representation $D_{M}^{L}\left(X_{+}^{p, q}\right)$ we use the following relations

$$
\begin{aligned}
J_{p}-\tilde{J}_{q} & =L+2 q+2 n \quad n=0,1,2, \ldots \\
M_{p} & =J_{p}, J_{p}-2, \ldots,-J_{p} \\
\tilde{M}_{q} & =M-M_{p}=\tilde{J}_{q}, \tilde{J}_{q}-2, \ldots,-\tilde{J}_{q},
\end{aligned}
$$

which follow from (2.20) and (2.23). These relations determine the set of all possible values of $J_{p}, \tilde{J}_{q}$ and $M_{p}$ for the given representation $D_{M}^{L}\left(X_{+}^{p, q}\right)$. An example can be seen in Fig. 1, which represents a section through the three-dimensional net with $M_{p}=$ const.

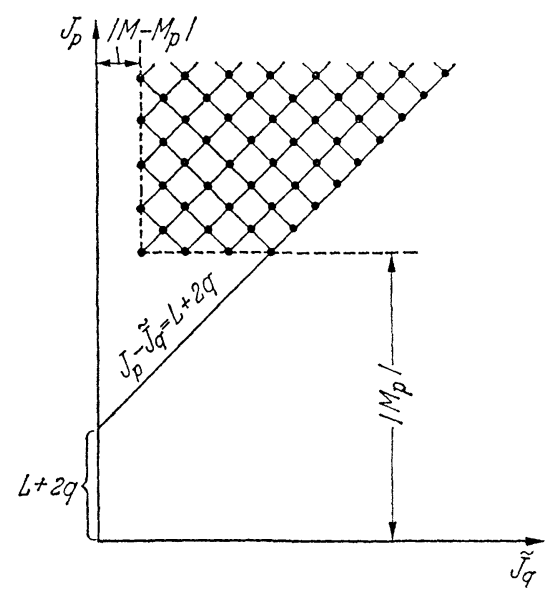

Fig. 1. An $M_{p}=$ const section through the three-dimensional net representing admissible values of $J_{p}, \tilde{J}_{q}$ and $M_{p}$ in a given representation $D_{M}^{L}\left(X_{+}^{p, q}\right), p \geqq q>1$

In a similar way, also, the structure of the Hilbert space $\mathscr{H}_{M}^{L_{M}}\left(X_{-,}^{p, q}\right)$ which corresponds to the representation $D_{M}^{L}\left(X^{p, q}\right)$, can be represented. The only difference lies in the fact that relation (2.29) (a) has to be replaced by

$$
\tilde{J}_{q}-J_{p}=L+2 p+2 n \quad n=0,1,2, \ldots .
$$

An example is given in Fig. 2, which represents a section through the corresponding net with $M_{p}=$ const.

\section{Discrete degenerate representations of the $U(p, 1)$ group}

In this case the homogeneous spaces creating the domains $X_{ \pm}^{p, 1}$ of the Hilbert spaces $\mathscr{H}_{M}^{L}\left(X_{ \pm}^{p, 1}\right)$ have the form

$$
X_{+}^{p, 1}=U(p, 1) / U(p-1,1) \text { and } X^{p, 1}=U(p, 1) / U(p)
$$

and can, again, be realized as the corresponding hypersurfaces given by equation (2.1) for $q=1$. The co-ordinate systems on these manifolds are 
introduced in the same way as in Section 2 and also the invariant operators $\Delta\left(X_{+}^{p}, 1\right)$ and $\hat{M}_{p+1}$ are obtained from those defined in Section 2 simply by putting $q=1$.

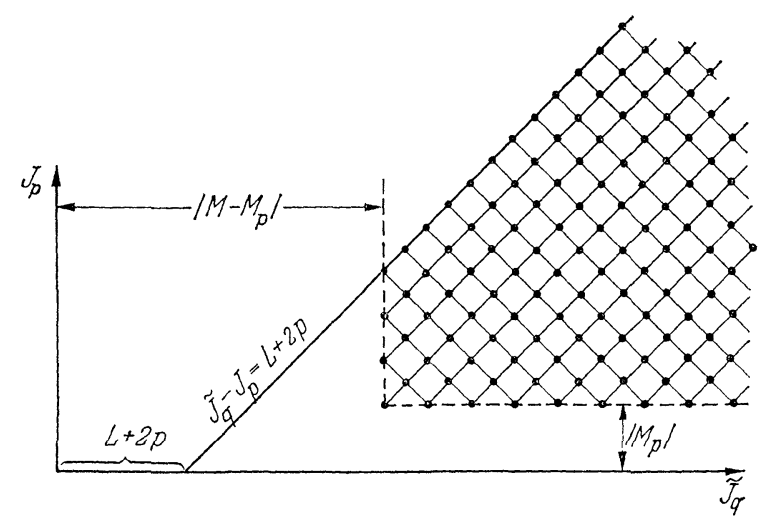

Fig. 2. An $M_{p}=$ const section through the three-dimensional net representing admissible values of $J_{p}, \tilde{J}_{q}$ and $M_{p}$ in a given representation $D_{M}^{L}\left(X^{p, q}\right), p \geqq q>1$

The Laplace-Beltrami operator $\Delta\left(X_{+}^{p, 1}\right), p>1$ has now the form $\Delta\left(X_{+}^{p, 1}\right)=$

$$
=-\frac{1}{\operatorname{ch}^{2 p-1} \theta \operatorname{sh} \theta} \frac{\partial}{\partial \theta} \operatorname{ch}^{2 p-1} \theta \operatorname{sh} \theta \frac{\partial}{\partial \theta}+\frac{\Delta\left(X^{p}\right)}{\operatorname{ch}^{2} \theta}-\frac{1}{\operatorname{sh}^{2} \theta} \frac{\partial^{2}}{(\partial \tilde{\varphi})^{2}}
$$

The essential difference from the previous case lies in the fact that the invariant second-order operator $\frac{\partial^{2}}{(\partial \tilde{\varphi})^{2}}$ of the $U(1)$ subgroup has eigenvalues $-\tilde{M}_{1}^{2}$ with $\tilde{M}_{1}$ an arbitrary integer. The set of differential equations for the representations on the manifold $X_{+}^{p, 1}$ is obtained from the set of equations (1.6), (1.7) by using (3.2) and

$$
\hat{M}_{p+1}=\hat{M}_{p}-i \frac{\partial}{\partial \tilde{\varphi}} \text {. }
$$

By the same procedure as before we reduce the eigenvalue problem of the operator (3.2) to the problem of finding the solution of the following equation

$$
\begin{array}{r}
\left(-\frac{1}{\operatorname{ch}^{2 p-1} \theta \operatorname{sh} \theta} \frac{d}{d \theta} \operatorname{ch}^{2 p-1} \theta \operatorname{sh} \theta \frac{d}{d \theta}-\frac{J_{p}\left(J_{p}+2 p-2\right)}{\operatorname{ch}^{2} \theta}+\frac{\tilde{M}_{1}^{2}}{\operatorname{sh}^{2} \theta}-\lambda\right) \\
\times \psi_{J_{p}\left|\tilde{M}_{1}\right|}^{\lambda}(\theta)=0 .
\end{array}
$$

The solution of (3.3) which is square integrable in the interval $\theta \in(0, \infty)$ with respect to the measure

$$
d \mu(\theta)=\operatorname{ch}^{2 p-1} \theta \operatorname{sh} \theta d \theta
$$


and regular at $\theta=0$ and $\theta \rightarrow \infty$ is now given by the function

$$
\begin{aligned}
& V_{J_{p}\left|\tilde{M}_{1}\right|}^{L}(\theta)=\frac{1}{\sqrt{N}} \operatorname{th}^{\left|\tilde{M}_{1}\right| \theta \mathrm{ch}^{-(L+2 p)} \theta \times} \\
& \quad \times{ }_{2} F_{1}\left(\frac{\left|\tilde{M}_{1}\right|-J_{p}+L}{2}+1, \frac{\left|\tilde{M}_{1}\right|+J_{p}+L}{2}+p ;\left|\tilde{M}_{1}\right|+1 ; \operatorname{th}^{2} \theta\right) \\
& N=\frac{\Gamma\left(\frac{J_{p}-\left|\tilde{M}_{1}\right|-L}{2}\right) \Gamma^{2}\left(\left|\tilde{M}_{1}\right|+1\right) \Gamma\left(\frac{L-\left|\tilde{M}_{1}\right|+J_{p}}{2}+p\right)}{2(L+p) \Gamma\left(\frac{J_{p}+\left|\tilde{M}_{1}\right|+L}{2}+p\right) \Gamma\left(\frac{J_{p}+\left|\tilde{M}_{1}\right|-L}{2}\right)}
\end{aligned}
$$

The invariant numbers $J_{p}$ and $\widetilde{M}_{1}$, which determine the irreducible unitary representations of the maximal compact subgroup $U(p) \times U(1)$, are restricted by the condition

$$
J_{p}-\left|\tilde{M}_{1}\right|=L+2+2 n, \quad n=0,1,2, \ldots .
$$

Consequently, the discrete spectrum of the operator $\Delta\left(X_{+}^{p, 1}\right)$ has the form

with

$$
\lambda=-L(L+2) \quad L=-p+1,-p+2, \ldots
$$

$$
L=J_{p}-\left|\tilde{M}_{1}\right|-2-2 n .
$$

In a definite representation with $L$ and $M$ fixed, the numbers $J_{p}$ and $\widetilde{M}_{1}$ can have only values which are restricted by the conditions

$$
\begin{array}{cl}
J_{p}-\left|\tilde{M}_{1}\right|=L+2+2 n & n=0,1,2, \ldots \\
J_{p}+\tilde{M}_{1}=M+2 n^{\prime} & n^{\prime}=0,1,2, \ldots J_{p} .
\end{array}
$$

As the value of $M_{p}$ is uniquely determined by the relation $M_{p}+\tilde{M}_{1}=M$, the structure of the corresponding Hilbert space can be now represented with the help of a two-dimensional net with the co-ordinates $J_{p}$ and $\tilde{M}_{1}$. One example of this net is given in Fig. 3. Note that for the definite $L$, the number $M$ can be an arbitrary integer having the same parity as $L$.

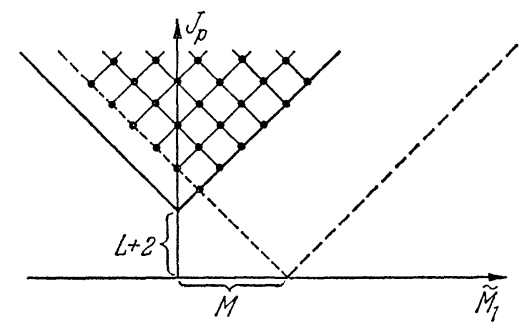

Fig. 3. Admissible values of $J_{p}$ and $\tilde{M}_{1}$ in a given representation $D_{M}^{L}\left(X_{+}^{p, 1}\right), p>1$

The basis of the representation space can be written in the form

$$
\mathbf{Y}_{M, M_{1}, \ldots M_{p}, \tilde{M}_{1}}^{L, J_{2}, \ldots J_{p}}\left(\Omega, \tilde{\phi}^{1}, \theta\right)=V_{J_{p}\left|\tilde{M}_{1}\right|}^{L}(\theta) \mathbf{Y}_{M_{1}, \ldots, M_{p}}^{J_{2}, \ldots, J_{p}}(\Omega) \frac{e^{i \tilde{M}_{1} \tilde{\phi}^{1}}}{\sqrt{2 \pi}}
$$

where the functions $\mathbf{Y}_{M_{1}, \ldots, M_{p}}^{J_{2}, \ldots, J_{p}}(\Omega)$ are given by formula (2.21). 
The representations acting on the Hilbert space $\mathscr{H}_{M}^{L}\left(X^{p, 1}\right), p>1$, are constructed in the same way. Instead of (3.7), we obtain now

$$
\begin{array}{cl}
\left|\tilde{M}_{1}\right|-J_{p}=L+2 p+2 n & n=0,1,2, \ldots \\
J_{p}+\tilde{M}_{1}=M+2 n^{\prime} & n^{\prime}=0,1,2, \ldots J_{p} .
\end{array}
$$

Two typical examples of spectra are given in Figs. 4 and 5. Let us mention that the spectrum of the invariant numbers $L$ and $M$ charac-

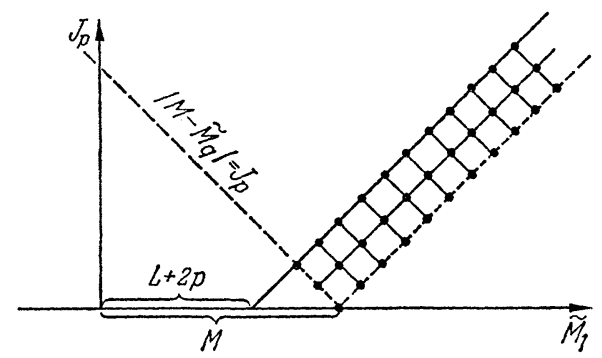

Fig. 4. Admissible values of $J_{p}$ and $\tilde{M}_{1}$ in a given representation $D_{M}^{L}\left(X^{p, 1}\right), p>1, M \geqq L+2 p$

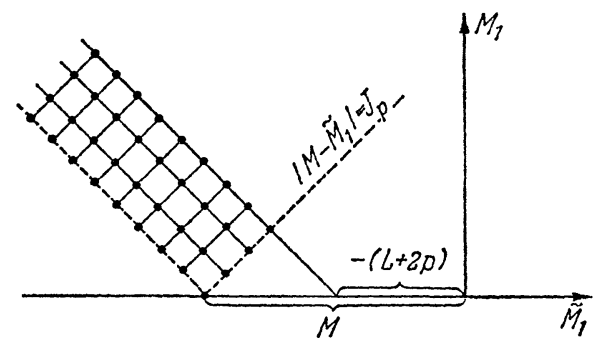

Fig. 5. Admissible values of $J_{p}$ and $\tilde{M}_{1}$ in a given representation $D_{M}^{L}\left(X^{p, 1}\right), p>1, M \leqq-(L+2 p)$

terizing the irreducible unitary representations $D_{M}^{L}\left(X_{\underline{p}, 1}\right)$ of the $U(p, 1)$ group $(p>1)$ is given by the conditions

$$
\begin{aligned}
& L=-p+1,-p+2, \ldots \\
& |M| \geqq L+2 p, \quad M \text { integer, } L+M \text { even . }
\end{aligned}
$$

In the case $p=q=1$ the differential equation as well as its solution is found by the same method. The representation is labelled by $L$ and $M$ and the conditions for $M_{1}$ and $\widetilde{M}_{1}$ in a definite representation are

$$
\begin{aligned}
\left|M_{1}\right|-\left|\tilde{M}_{1}\right| & =L+2+2 n \quad n=0,1,2, \ldots \\
M_{1}+\tilde{M}_{1} & =M .
\end{aligned}
$$

The "net" of subspaces $\mathscr{H}_{M}^{L} M_{1} \tilde{M}_{1}\left(X_{+}^{1,1}\right)$ of the space $\mathscr{H}_{M}^{L}\left(X_{+}^{1}, 1\right)$ reduces here to a string of points. The spectrum of the invariant numbers $L$ and 
$M$ is

$$
\begin{aligned}
& L=0,1,2, \ldots \\
& |M| \geqq L+2, \quad M \text { integer, } L+M \text { even . }
\end{aligned}
$$

Two typical examples of representations $D_{M}^{L}\left(X_{+}^{1,1}\right)$ are shown in Fig. 6.

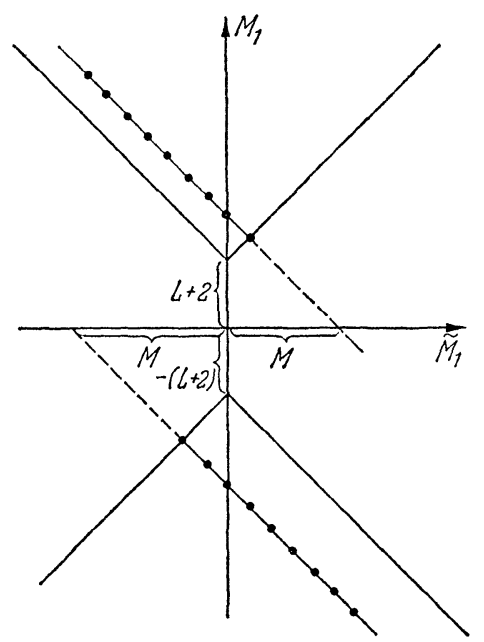

Fig. 6. Two examples of one-dimensional "nets" representing admissible values of $M_{1}$ and $\tilde{M}_{1}$ in the representations $D_{M}^{L}\left(X_{+}^{1,1}\right)$. One example is for $M \geqq L+2$, the other for $M \leqq-(L+2)$

Since the Hilbert spaces $\mathscr{H}_{M}^{L}\left(X_{+}^{1,1}\right)$ and $\mathscr{H}_{M}^{L}\left(X_{-}^{1,1}\right)$ are identical, the corresponding representations are equivalent and are obtained from one another by exchanging the role of $M_{1}$ and $\tilde{M}_{1}$.

\section{Irreducibility and unitarity}

The Lie algebra $R_{p, q}$ of the $U(p, q)$ group consists of $(p+q)^{2}$ basic elements; if the basis is suitably chosen one can distinguish the following different types of basic elements:

1) $p+q$ operators $L_{i i}^{-}(i=1,2, \ldots p+q)$ belonging to a Cartan subalgebra of $R_{p, q}$.

2) $\left(\begin{array}{l}p \\ 2\end{array}\right)+\left(\begin{array}{c}q \\ 2\end{array}\right)$ operators $L_{i j}^{+}(i, j=1,2, \ldots p$ or $i, j=p+1, p+2, \ldots$ $p+q, i<j)$ which correspond to the subgroup $S O(p) \times S O(q)$ of $U(p, q)$.

3) $\left(\begin{array}{c}p \\ 2\end{array}\right)+\left(\begin{array}{l}q \\ 2\end{array}\right)$ operators $L_{i j}^{-}(i, j=1,2, \ldots p$ or $i, j=p+1, p+2, \ldots$ $p+q, i<j)$ which, together with $L_{i}$ and $L_{i j}^{+}$create the subalgebra belonging to the maximal compact subgroup $U(p) \times U(q)$ of $U(p, q)$.

4) $2 p q$ non-compact operators $B_{i j}^{+}, B_{i j}^{-}$where $i=1,2, \ldots p$ $j=p+1, \ldots p+q$. 

form:

The commutation relations among these operators have the following

$$
\begin{aligned}
& {\left[L_{i j}^{+}, L_{k l}^{ \pm}\right]= \pm \delta_{k j} L_{i l}^{ \pm} \pm \delta_{i l} L_{k j}^{+}-\delta_{k i} L_{j l}^{+}+\delta_{j l} L_{k i}^{+}} \\
& {\left[L_{i j}^{ \pm}, B_{k l}^{ \pm}\right]= \pm \delta_{k j} B_{i l}^{+} \mp \delta_{i l} B_{k j}^{+}-\delta_{k i} B_{j l}^{+}+\delta_{j l} B_{k i}^{+}} \\
& {\left[L_{i j}^{ \pm}, B_{k l}^{\mp}\right]=\delta_{k j} B_{i l}^{-}-\delta_{i l} B_{k j}^{-} \mp \delta_{k i} B_{j l}^{-} \pm \delta_{j l} B_{k i}^{-}} \\
& {\left[B_{i j}^{+}, B_{k l}^{ \pm}\right]= \pm \delta_{k j} L_{i l}^{+} \pm \delta_{i l} L_{k j}^{+}+\delta_{k i} L_{j l}^{+}-\delta_{j l} L_{i k i}^{+}} \\
& L_{j i}^{ \pm} \equiv \mp L_{i j}^{ \pm}, B_{j i}^{ \pm} \equiv \pm B_{i j}^{ \pm},
\end{aligned}
$$

no summation.

Due to the fact that the algebra $R_{p, q}$ of $U(p, q)$ is a subalgebra of the algebra of the $S O(2 p, 2 q)$ group we can express our basis of $R_{p, q}$ in the form of certain linear combinations of the generators of $S O(2 p, 2 q)$. By an explicit calculation we can see that

$$
\begin{aligned}
& \left.L_{i j}^{+}=L_{2 i-1,2 j-1}+L_{2 i, 2 j}\right) i, j=1,2, \ldots p, \quad i<j \quad \text { or } \\
& \left.L_{i j}^{-}=L_{2 i-1,2 j}-L_{2,2 j-1}\right\} i, j=p+1, \ldots p+q, \quad i<j \\
& L_{i i}^{-}=2 L_{2 i-1,2 i} \quad i=1,2, \ldots p+q \\
& \left.B_{i j}^{+}=B_{2 i-1,2 j-1}+B_{2 i, 2 j}\right\} i=1,2, \ldots p \\
& \left.B_{i j}^{-}=B_{2 i-1,2 j}-B_{2 i, 2 j-1}\right\} j=p+1, \ldots p+q
\end{aligned}
$$

where $L_{k l}(k, l=1,2, \ldots 2 p$ or $k, l=2 p+1, \ldots 2(p+q))$ and $B_{k l}$ $(k=1,2, \ldots 2 p, l=2 p+1, \ldots 2(p+q))$ are the generators of the compact and non compact rotations of the $S O(2 p, 2 q)$ group respectively.

In order to calculate the action of the generators of the $U(p, q)$ group on the set of harmonic functions (2.18) or (3.8) we represent the Lie algebra $R_{p, q}$ by the Lie algebra of the operators of differentiation with respect to the co-ordinates $\varphi^{1}, \ldots, \varphi^{p}, \tilde{\varphi}^{1}, \ldots \tilde{\varphi}^{q}, \vartheta^{2}, \ldots \vartheta^{p}, \tilde{\vartheta}^{2}, \ldots \tilde{\vartheta}^{q}$ and $\theta$. This representation of the Lie algebra $R_{p, q}$ will be denoted by $\widetilde{R}_{p, q}$. We give here the explicit form of two basic operators of $\widetilde{R}_{p, q}$, which will be useful in the following.

$$
\begin{aligned}
B_{p, p+q}^{+} \pm i B_{p, p+q}^{-}=e^{ \pm i\left(\phi^{p}-\tilde{\varphi}^{q}\right)}\left[\cos \vartheta^{p} \cos \tilde{\vartheta^{q}} \frac{\partial}{\partial \theta}-\right. \\
-\operatorname{cth} \theta \cos \vartheta^{p}\left(\sin \tilde{\vartheta}^{q} \frac{\partial}{\partial \tilde{\vartheta}^{q}} \pm i \frac{1}{\cos \tilde{\vartheta}^{q}} \frac{\partial}{\partial \tilde{\varphi}^{q}}\right)- \\
\left.-\operatorname{th} \theta \cos \tilde{\vartheta}^{q}\left(\sin \vartheta^{p} \frac{\partial}{\partial \vartheta^{p}} \mp i \frac{1}{\cos \vartheta^{p}} \frac{\partial}{\delta \varphi^{p}}\right)\right]
\end{aligned}
$$

\section{Irreducibility}

To prove the irreducibility of our representation $D_{M}^{L}\left(X_{+}^{p, q}\right)$ on the Hilbert space $\mathscr{H}_{M}^{L}\left(X_{+}^{p, q}\right)$ we show that $\mathscr{H}_{M}^{L}\left(X_{+}^{p, q}\right)$ contains no subspace 
invariant with respect to the action of the Lie algebra $\widetilde{R}_{p, q}$. The structure of $\mathscr{H}_{M}^{L_{M}}\left(X_{+}^{p, q}\right)$ is given by formula (2.28).

From the proof of irreducibility given in the Appendix it follows that our representation of the algebra of the maximal compact subgroup $U(p) \times U(q)$ is irreducible in the space $\mathscr{H}_{M M_{p}}^{L J_{p} \tilde{J}_{q_{q}}} \tilde{M}_{q}$. Thus, to perform the proof of irreducibility in the non-compact case, it is sufficient to show

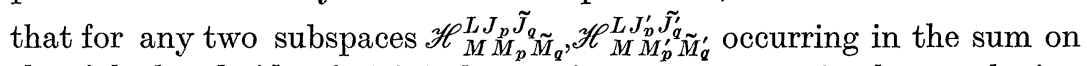
the right-hand side of (2.28) there exists an operator in the enveloping algebra of $\widetilde{R}_{p, q}$ such that transforms one element of $\mathscr{H}_{M M_{p}}^{L J_{p} \widetilde{J}_{q} \widetilde{M}_{q}}$ into an element of $\mathscr{H}_{M}^{L J_{j}^{\prime} \tilde{J}_{\rho}^{\prime}} \tilde{M}_{\dot{q}}^{\prime}$.

1. The case $p \geqq q>1$. The structure (2.28) of $\mathscr{H}_{M}^{L}\left(X_{+}^{p, q}\right)$ can be represented with the help of a three-dimensional net with co-ordinates $J_{p}, \tilde{J}_{q}$ and $M_{p}$, in which each knot denotes one subspace $\mathscr{H}_{M M_{p}}^{L J_{j} \tilde{q}_{q} \tilde{M}_{q}}$ of $\mathscr{H}_{M}^{L}$ (see Fig. 1). To prove the irreducibility it is sufficient to find some operators $B_{i} \in \widetilde{R}_{p, q}$ and one harmonic function $\mathbf{Y}_{M M_{1} \ldots M_{p}}^{L J_{2} \ldots J_{p}{\widetilde{M_{2}}}_{1} \ldots{\tilde{J_{q}}}_{1} \ldots \tilde{M}_{q}}$ such that the operators $B_{i}$ can perform the transition into all nearest neighbouring knots of the net, i.e., into the Hilbert spaces $\mathscr{H}_{M M_{p}^{\prime}}^{L J_{p}^{\prime} \tilde{J}_{\dot{q}}^{\prime}} \tilde{M}_{q}^{\prime}$ with $J_{p}^{\prime}=J_{p} \pm 1, \tilde{J}_{q}^{\prime}=\tilde{J}_{q} \pm 1$ and $M_{p}^{\prime}=M_{p} \pm 1$. We follow here the method which has been developed in [8].

By an explicit calculation we can see that the operators

$$
B_{1} \equiv B_{p, p+q}^{+}+i B_{p, p+q}^{-} \quad \text { and } \quad B_{2} \equiv B_{p, p+q}^{+}-i B_{p, p+q}^{-}
$$

given by formula (4.4) have the desired properties, $B_{1}$ performing the four steps $J_{p}^{\prime}=J_{p} \pm 1, \tilde{J}_{q}^{\prime}=\tilde{J}_{q} \pm 1, M_{p}^{\prime}=M_{p}+1$ and $B_{2}$ performing the other four steps $J_{p}^{\prime}=J_{p} \pm 1, \tilde{J}_{q}^{\prime}=\tilde{J}_{q}^{p} \pm 1, M_{p}^{\prime}=M_{p}-1$ if acting on

$$
\mathbf{Y}_{M M_{p}}^{L J_{p} \tilde{J_{q}}} \tilde{M}_{q} \equiv \mathbf{Y}_{M, 0, \ldots 0, M_{p}, 0, \ldots, 0, \tilde{M}_{q}}^{L, 0, \ldots, J_{p} 0, \ldots, \tilde{J_{\alpha}}}(\Omega, \widetilde{\Omega}, \theta) .
$$

Performing the calculation we obtain the following result:

$$
\begin{aligned}
& B_{1} \mathbf{Y}_{M, M_{p}, \tilde{M}_{q}}^{L, J_{p}, \tilde{I}_{\tilde{I}}}=-\sqrt{\left(J+\tilde{J}+1+L^{\prime}\right)\left(J+\tilde{J}+1-L^{\prime}\right)} \times \\
& \times a_{+}^{+} \tilde{a} \pm \mathbf{Y}_{M, M_{p}+1, \tilde{M}_{q}-1}^{L, J_{p}+1, \tilde{J_{q}}+1}+ \\
& +\sqrt{\left(J-\tilde{J}+1+L^{\prime}\right)\left(\overline{\left.J-\tilde{J}+1-L^{\prime}\right)}\right.} a_{+}^{+} \tilde{a}_{-}^{-} \mathbf{Y}_{M, M_{p}+1, \tilde{M}_{q}-1}^{L, J_{p}+1, \tilde{q}_{q}-1}-
\end{aligned}
$$

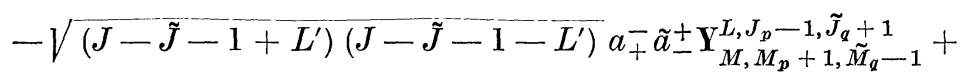

$$
\begin{aligned}
& +\sqrt{\left(J+\tilde{J}-1+L^{\prime}\right)\left(J+\tilde{J}-1-L^{\prime}\right)} a_{+}^{-} \tilde{a}_{-}^{-} \mathbf{Y}_{M, M_{p}+1, \tilde{M}_{q}-1}^{L, J_{p}-1, \tilde{J}_{q}-1}
\end{aligned}
$$

where

$$
J=J_{p}+p-1, \quad \tilde{J}=\tilde{J}_{q}+q-1, \quad L^{\prime}=L+p+q-1
$$


and

$$
\begin{gathered}
a_{+}^{+} \equiv a_{+}^{+}\left(J, M_{p}, p\right)=\frac{1}{2} \frac{\sqrt{J+M_{p}+p-1} \sqrt{J+M_{p}-p+3}}{\sqrt{J(J+1)}} \\
a_{+}^{-} \equiv a_{+}^{-}\left(J, M_{p}, p\right)=\frac{1}{2} \frac{\sqrt{J-M_{p}-p+1} \sqrt{J-M_{p}+p-3}}{\sqrt{J(J-1)}} \\
a_{ \pm}^{ \pm}\left(J, M_{p}, p\right)=a_{+}^{ \pm}\left(J,-M_{p}, p\right) \\
\tilde{a}_{ \pm}^{ \pm} \equiv a_{ \pm}^{ \pm}\left(\tilde{J}, \tilde{M}_{q}, q\right) .
\end{gathered}
$$

The corresponding expression for $B_{2} \mathbf{Y}_{M, M_{p}, \tilde{M}_{q}}^{L, J_{p}, \tilde{J}_{q}}$ can be obtained from (4.6) by performing the following changes:

$$
\begin{aligned}
& \mathbf{Y}_{M, M_{p}+1, \tilde{M}_{q}-1}^{L, J_{p}^{\prime}, \tilde{J}_{q}^{\prime}} \rightarrow \mathbf{Y}_{M, M_{p}-1, \tilde{M}_{q}+1}^{L, J_{J_{p}^{\prime}}^{\prime} \tilde{J}_{q}^{\prime}} \quad \text { for } \quad J_{p}^{\prime}=J_{p} \pm 1 \\
& \tilde{J}_{q}^{\prime}=\tilde{J}_{q} \pm 1 \\
& a_{+}^{ \pm} \rightarrow a_{ \pm}^{ \pm} \\
& \tilde{a}_{ \pm}^{ \pm} \rightarrow \tilde{a}_{+}^{ \pm} \text {. }
\end{aligned}
$$

By a successive application of the operators $B_{p, p+q}^{+} \pm i B_{p, p+q}^{-}$ every point of the given diagram can be reached starting from an arbitrary point.

The proof of the irreducibility of the representation $D_{M}^{L}\left(X^{p, q}\right)$ for $p \geqq q>1$ is analogous.

2. The case $p>q=1$. The decomposition (2.28) of the space $\mathscr{H}_{M}^{L}\left(X_{+}^{p, 1}\right)$ reduces to a double sum an can be represented with the help of a two-dimensional net (see Fig. 3). The operators $B_{1}$ and $B_{2}$ have, according to (4.4), the following form

$$
\begin{aligned}
B_{p p+1}^{+} \pm i B_{p p+1}^{-}= & e^{ \pm i(\varphi p-\tilde{\phi})}\left[\cos \vartheta^{p} \frac{\partial}{\partial \theta} \mp i \operatorname{cth} \theta \cos \vartheta^{p} \frac{\partial}{\partial \tilde{\phi}}-\right. \\
& \left.-\operatorname{th} \theta\left(\sin \vartheta^{p} \frac{\partial}{\partial \vartheta^{p}} \mp \frac{i}{\cos \vartheta^{p}} \frac{\partial}{\partial \varphi^{p}}\right)\right], \tilde{\phi} \equiv \tilde{\varphi}^{1}
\end{aligned}
$$

and we let them act on (see (3.8))

$$
\mathbf{Y}_{M, M_{p}, \tilde{M}_{1}}^{L, J_{p}} \equiv \mathbf{Y}_{0, \ldots 0, M_{p}}^{0, \ldots, J_{p}}(\Omega) \frac{e^{i \tilde{M_{1}} \tilde{\phi}}}{\sqrt{2 \pi}} V_{J_{p},\left|\tilde{M}_{1}\right|}^{L}(\theta) .
$$

Using the same procedure we obtain

$$
\begin{aligned}
& B_{1} \mathbf{Y}_{M, M_{p}, \tilde{M}_{1}}^{L, J_{p}}=\sqrt{\left(J-\tilde{M}_{1}+1+L^{\prime}\right)\left(J-\tilde{M}_{1}+1-L^{\prime}\right)} \times \\
& \times a_{+}^{+} \mathbf{Y}_{M, M_{p}+1, \tilde{M}_{1}-1}^{L, J_{p}+1} \\
& +\sqrt{\left(J+\tilde{M}_{1}-1+L^{\prime}\right)\left(J+\tilde{M}_{1}-1-L^{\prime}\right)} a_{+}^{-} \mathbf{Y}_{M, M_{p}+1, \tilde{M}_{1}-1}^{L, J_{p}-1} .
\end{aligned}
$$

The action of $B_{2}$ is obtained from (4.11) if all symbols (except for $\tilde{J}$ and $\tilde{a}_{ \pm}^{ \pm}$, which do not occur here) are changed according to (4.9). 
A successive application of the operators $B_{1}$ and $B_{2}$ makes it possible to perform any transition from point to point within the given diagram.

The irreducibility of the representation $D_{M}^{L}\left(X^{p, 1}\right)$ is proved analogously.

3. The case $p=q=1$. The sum in (2.28) is now one-dimensional (over $M_{1}, M_{1}+\tilde{M}_{1}=M$ ) and the operators $B_{1}$ and $B_{2}$ reduce to

$B^{+} \pm i B^{-}=e^{ \pm i(\phi-\tilde{\phi})}\left[\frac{\partial}{\partial \theta} \mp i \operatorname{cth} \theta \frac{\partial}{\partial \tilde{\phi}} \pm i \operatorname{th} \theta \frac{\partial}{\partial \phi}\right]$

$$
\phi \equiv \varphi^{1}, \tilde{\phi} \equiv \tilde{\varphi}^{1} .
$$

The basic vectors have the form

$$
\mathbf{Y}_{M, \tilde{M}_{1}, M_{1}}^{L}(\phi \tilde{\phi} \theta)=\frac{1}{2 \pi} e^{i M_{1} \phi} e^{i \tilde{M}_{1} \tilde{\phi}} V_{\left|M_{1}\right|,\left|\tilde{M}_{1}\right|}^{L}(\theta) .
$$

We obtain

$$
\begin{aligned}
B_{1} \mathbf{Y}_{M, M_{1}, \tilde{M}_{1}}^{L}=\sqrt{\left(M_{1}-\tilde{M}_{1}+1+L^{\prime}\right)\left(M_{1}-\tilde{M}_{1}+\right.} & \left.1-L^{\prime}\right) \\
\times & \times \mathbf{Y}_{M, M_{1}+1, \tilde{M}_{1}-1}^{L}
\end{aligned}
$$

and

$$
\begin{aligned}
& B_{2} \mathbf{Y}_{M, M_{1}, \tilde{M}_{1}}^{L}=-\sqrt{\left(\tilde{M}_{1}-M_{1}+1+L^{\prime}\right)\left(\tilde{M}_{1}-M_{1}+1-L^{\prime}\right)} \times \\
& \times \mathbf{Y}_{M, M_{1}-1, \tilde{M}_{1}+1}^{L} .
\end{aligned}
$$

So, the irreducibility is proved also in this case. We can see from (4.15) that the factor $\left|\tilde{M}_{1}\right|-\left|M_{1}\right|+L+2$ stops the raising of $M_{1}$ at the point

$$
-M_{1}+\tilde{M}_{1}=L+2 \quad M_{1}<0 \quad \tilde{M}_{1} \leqq 0
$$

as it is prescribed in the summation (2.28) for this case (see (3.11)). The same factor stops also the lowering of $M_{1}$ at the point

$$
M_{1}-\tilde{M}_{1}=L+2 \quad M_{1}>0 \quad \tilde{M}_{1} \geqq 0
$$

(see (4.16)), in agreement with the condition (3.11).

\section{Unitarity}

An element $g$ of the $U(p, q)$ group and its representation $T_{g}$ in the Hilbert space $\mathscr{H}_{M}^{L}\left(X_{+}^{p, q}\right)$ are connected by the relation

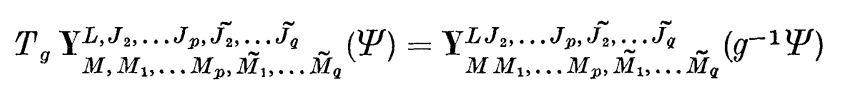

where $\Psi$ means a point $(\Omega, \widetilde{\Omega}, \theta)$ of the manifold $X_{+}^{p, q}$ and $g^{-1} \Psi$ means its left translation. As the group $U(p, q)$ acts transitively on $X_{+}^{p, q}$, the unitarity follows from the left invariance of the measure $d \mu\left(X_{+}^{p, q}\right)$ on $X_{+}^{p, q}$.

The unitarity of the representation $D_{M}^{L}\left(X^{p} \underline{q}\right)$ is proved by the same argument.

18 Commun. math. Phys., Vol. 3 


\section{Conclusion}

We have shown that for any non-compact unitary group $U(p, q)$, $p \geqq q$ there exist two different discrete series of degenerate representations, $D_{M}^{L}\left(X_{+}^{p, q}\right)$ and $D_{M}^{L}\left(X^{p, q}\right)$, which coincide only for $p=q$. These representations are characterized by only two numbers, $L$ and $M, L=-(p+q)+$ $+2,-(p+q)+3, \ldots, M$ being an integer having the same parity as $L$. In the cases $D_{M}^{L}\left(X_{\underline{p}, 1}\right)$ and $D_{M}^{L}\left(X^{1,1}\right)$ the values of $L, M$ are restricted by additional conditions (3.10) and (3.12) respectively.

As $D_{M}^{L}\left(X_{+}^{p, q}\right)$ and $D_{M}^{L}\left(X^{p, q}\right)$ are the degenerate representations (see Footnote 3 ), the corresponding maximal set of commuting operators is extremely small, consisting of only the $2(p+q)-1$ independent operators $^{4}$ for which we can choose invariant operators $\Delta\left(X^{p, q}\right)$ and $\hat{M}_{p+q} \equiv \hat{M}$ of the $U(p, q)$ group and the sets $C_{p}$, $\widetilde{C}_{q}$ of commuting operators of the maximal compact subgroup $U(p) \times U(q)$ given by ${ }^{5}$

$$
\begin{aligned}
& C_{p} \equiv\left\{\Delta\left(X^{p}\right), \hat{M}_{p}, \Delta\left(X^{p-1}\right), \hat{M}_{p-1}, \ldots \Delta\left(X^{2}\right), \hat{M}_{2}, \hat{M}_{1}\right\} \\
& \widetilde{C}_{q} \equiv\left\{\widetilde{\Delta}\left(X^{q}\right), \hat{\widetilde{M}}_{q}, \widetilde{\Delta}\left(X^{q-1}\right), \hat{\mathscr{M}}_{q-1}, \ldots \widetilde{\Delta}\left(X^{2}\right), \hat{\widetilde{M}}^{2}, \hat{M}_{1}\right\} .
\end{aligned}
$$

All these operators commute with the parity operator $\hat{P}$ defined by $\hat{P} z^{i}=-z^{i}, i=1,2, \ldots p+q$. For every irreducible representation defined by $L$ and $M$ the parity is equal to the parity of $L$.

Our approach made it possible to obtain rather easily the sets of orthonormal functions $\mathbf{Y}_{M, M_{1}, \ldots, M_{p}, \tilde{M}_{1}, \ldots \tilde{M}_{q}}^{L, J_{2}, \ldots, J_{p}, \tilde{J}_{2}}(\Omega, \widetilde{\Omega})$ which create the bases of the representation spaces. This is important for physical applications since the explicit knowledge of the basis functions plays a great role.

The representations $D_{M}^{L}\left(X_{+}^{p, q}\right)$ and $D_{M}^{L}\left(X^{p, q}\right)$ themselves seem to us to be of special interest from the point of view of applications in elementary particle physics, for the following reasons:

(i) The maximal set $C \equiv\left\{\Delta\left(X^{p, q}\right), C_{p}, \widetilde{C}_{q}\right\}$ of commuting operators contains a very small number of elements. The set of numbers $L$, $M_{1}, \ldots M_{p}, J_{2}, \ldots J_{p}, \tilde{M}_{1}, \ldots \tilde{M}_{q}, \tilde{J}_{2}, \ldots \tilde{J}_{q}$ which label each basic function determines the eigenvalues of the maximal set $C$ (see (2.16) and (A.6)) and may be related to a set of quantum numbers characterising a given physical state. The small number of elements of $C$ is important due to the fact that in physical applications unitary groups with comparatively high values of $p$ or $p$ and $q$ have been used.

(ii) The spectra of all quantum numbers $L, M_{1}, \ldots M_{p}, J_{2}, \ldots J_{p}$, $\tilde{M}_{1}, \ldots \tilde{M}_{q}, \tilde{J}_{2}, \ldots \tilde{J}_{q}$ are discrete.

4 For the principal non-degenerate representation the number of commuting operators whose eigenvalues label the basic functions is $\frac{1}{2}(p+q)(p+q+1)$.

5 Note that $\hat{M}_{p}+\hat{\widetilde{M}}_{q}=\hat{M}$. 
(iii) The biharmonic co-ordinate system, which we have used to parametrize the manifolds $X_{ \pm}^{p, q}$, leads to the maximal number of linear operators $\hat{M}_{1}, \ldots \hat{M}_{p}, \hat{M}_{1}, \ldots \hat{M}_{q}$. Naturally, if we interpret them physically, we obtain the maximal set of additive quantum numbers.

Acknowledgments. The authors would like to thank Professors ABDUs SALAM and PAOLO BUdINI and the IAEA for the hospitality kindly extended to them at the International Centre for Theoretical Physics, Trieste, as well as to Professors Abdus Salam and Paolo Budini for valuable discussions.

It is also a pleasure to thank Drs. N. LIMIĆ and J. NIEDERLE for interesting comments and discussions.

\section{Appendix}

\section{Degenerate representations of compact unitary groups $U(p)$}

\section{The basic functions}

For an arbitrary compact unitary group $U(p)$ there exists a series of degenerate unitary representations which are created on the finitedimensional Hilbert spaces $\mathscr{H}_{M_{p}}^{J_{p}}$ of functions with the domain given by the manifold $X$

$$
X^{p}=U(p) / U(p-1) .
$$

This manifold represents by itself the symmetric Cartan space, on which the group $U(p)$ acts transitively.

As a model of $X^{p}$ we choose the hypersurface in the $p$-dimensional complex space $C$ given by the equation

$$
z^{1} \bar{z}^{1}+z^{2} \bar{z}^{2}+\cdots+z^{p} \bar{z}^{p}=1 .
$$

The co-ordinate system on $X^{p}$ is created in a recursive manner described by formula (2.4) and the corresponding metric tensor is given by formula (2.7) for $q=0$. The Laplace-Beltrami operator $\Delta\left(X^{p}\right)$ is then expressed by a recursion formula in terms of $\Delta\left(X^{p-1}\right)$ in the following form (see [7]):

$$
\begin{aligned}
\Delta\left(X^{p}\right)=\frac{1}{\sin ^{2 p-3} \vartheta^{p} \cos \vartheta^{p}} \frac{\partial}{\partial \vartheta^{p}} & \sin ^{2 p-3} \vartheta^{p} \cos \vartheta^{p} \frac{\partial}{\partial \vartheta^{p}}+ \\
& \quad+\frac{1}{\cos ^{2} \vartheta^{p}} \frac{\partial^{2}}{\left(\partial \varphi^{p}\right)^{2}}+\frac{1}{\sin ^{2} \vartheta^{p}} \Delta\left(X^{p-1}\right)
\end{aligned}
$$

Further, the invariant first-order operator $\hat{M}$ belonging to the centre of the $U(p)$-algebra has the form

$$
\hat{M}_{p}=-i \sum_{i=1}^{p} \frac{\partial}{\partial \varphi^{i}}=-i \frac{\partial}{\partial \varphi^{p}}+\hat{M}_{p-1} .
$$

If we express the eigenfunctions of the operators (A.2) and (A.3) in the form of a product of eigenfunctions of $\Delta\left(X^{p-1}\right)$ and $\hat{M}_{p-1}$ and an 
unknown function $\psi_{m_{p}}^{J_{p}, J_{p}-1}\left(\vartheta^{p}\right) \cdot f_{m_{p}}\left(\varphi^{p}\right)$ we obtain the general solution of the eigenvalue problem in the form of functions each of which depends only on one angle, $\varphi^{i}$ or $\vartheta^{k}$. The solution of the equation for $\psi_{m_{p}}^{J_{p}, J_{p}-1}\left(\vartheta^{p}\right)$ has the form

$$
\begin{aligned}
\psi_{m_{p}}^{J_{p}, J_{p}-1}\left(\vartheta^{p}\right)= & \operatorname{tg}\left|J_{p-1}\right| \vartheta^{p} \cdot \cos ^{J_{p}} \vartheta^{p}{ }_{2} F_{1}\left[\frac{1}{2}\left(\left|J_{p-1}\right|-J_{p}+m_{p}\right)\right. \\
& \left.\left.\frac{1}{2}\left(\left|J_{p-1}\right|-J_{p}-m_{p}\right) ;\left|J_{p-1}\right|+p-1 ;-\operatorname{tg}^{2} \vartheta^{p}\right)\right]
\end{aligned}
$$

where $J_{p}, J_{p-1}$ and $m_{p}$ are restricted by the condition that ${ }_{2} F_{1}$ be a polynomial and $\psi$ be square integrable:

$$
\left|J_{p-1}\right|+\left|m_{p}\right|=J_{p}-2 n \quad n=0,1,2, \ldots .
$$

The eigenvalues $\lambda_{p}$ of $\Delta\left(X^{p}\right)$ have the form

$$
\lambda_{p}=-J_{p}\left(J_{p}+2 p-2\right) \text {. }
$$

Further, the eigenvalue equation of the operator $\hat{M}_{p}$ leads to the following relation among $m_{1}, m_{2}, \ldots m_{p}$ and the eigenvalue $M_{p}$ of $\hat{M}_{p}$ :

$$
M_{p}=\sum_{i=1}^{p} m_{i} \text {. }
$$

Under the condition (A.5), the function $\psi_{m_{p}}^{J_{p}, J_{p}-1}\left(\vartheta^{p}\right)$ can be expressed in terms of $d$-functions which appear in the harmonic analysis of the threedimensional rotation group. The set of eigenfunctions of the operators $\Delta\left(X^{p}\right)$ and $\hat{M}_{p}$ can be written in the following manner:

$$
Y_{m_{1}, \ldots, m_{p}}^{J_{2}, \ldots, J_{p}}(\omega)=\frac{1}{\sqrt{N_{p}^{\prime}}} \prod_{l=1}^{p} \exp \left(i m_{l} \varphi^{l}\right) \cdot \prod_{k=2}^{p} \sin ^{2-k} \vartheta^{k} \cdot d_{\alpha_{k}, \beta_{k}}^{\frac{1}{2}\left(J_{k}+k-2\right)}\left(2 \vartheta^{k}\right)
$$

where

$$
\begin{array}{cl}
\alpha_{k}=\frac{1}{2}\left(m_{k}+J_{k-1}+k-2\right) & k=2, \ldots, p ; \quad J_{1} \equiv m_{1} \\
\beta_{k}=\frac{1}{2}\left(m_{k}-J_{k-1}-k+2\right) & \\
\omega \equiv\left\{\varphi^{1}, \varphi^{2}, \ldots, \varphi^{p}, \vartheta^{2}, \ldots, \vartheta^{p}\right\} & 0 \leqq \varphi^{i} \leqq 2 \pi \quad i=1,2, \ldots, p \\
& 0 \leqq \vartheta^{k} \leqq \frac{\pi}{2} \quad k=2,3, \ldots, p
\end{array}
$$

and

$$
N_{p}^{\prime}=2 \pi^{p} \prod_{k=2}^{p} \frac{1}{J_{k}+k-1}
$$

The functions $Y_{m_{1}, \ldots, m_{p}}^{J_{2}, \ldots, J_{p}}(\omega)$ create an orthonormal set of functions on the manifold $X^{p}$ with respect to the measure

$$
\begin{aligned}
d \mu\left(X^{p}\right)=\sqrt{\left|\bar{g}\left(X^{p}\right)\right|} \prod_{l=1}^{p} d \varphi^{l} \prod_{k=2}^{p} d \vartheta^{k} & = \\
& =\prod_{k=2}^{p} \sin ^{2 k-3} \vartheta^{k} \cos \vartheta^{k} d \vartheta^{k} \prod_{l=1}^{p} d \varphi^{l}
\end{aligned}
$$


For a closer discussion of representations of the $U(p)$ and $S U(p)$ groups it is useful to introduce new azimuthal angles $\phi^{1}, \ldots, \phi^{p}$ which are defined by

and

$$
\phi^{k}=\varphi^{k}-\varphi^{k+1} \quad k=1,2, \ldots, p-1
$$

$$
\phi^{p}=\sum_{i=1}^{p} \varphi^{i}
$$

i.e.

$$
\begin{aligned}
\varphi^{l} & =\frac{1}{p}\left(\phi^{p}+\sum_{i=1}^{p-1}(p-i) \phi^{i}\right)-\sum_{i=1}^{l-1} \phi^{i} \\
l & =1,2, \ldots, p .
\end{aligned}
$$

The restrictions $0 \leqq \phi^{l} \leqq 2 \pi, l=1,2, \ldots p$ on the angles $\varphi^{l}$ impose the following restrictions on $\phi^{l}:^{6}$

$$
\begin{aligned}
-2 \pi & \leqq \phi^{1} \leqq 2 \pi \\
-2 \pi & \leqq \phi^{p-1} \leqq 2 \pi \\
0 & \leqq \phi^{p} \leqq 2 \pi p .
\end{aligned}
$$

Further, it is convenient to introduce, instead of $m_{1}, \ldots m_{p}$, the numbers $M_{l}$,

$$
M_{l}=\sum_{i=1}^{l} m_{i} \quad l=1,2, \ldots, p .
$$

A given representation of the $U(p)$ group is characterized by the numbers $J_{p}$ and $M_{p}$, which, due to (A.5) and (A.7), fulfil the following restrictive conditions:

$$
\begin{aligned}
J_{p} & =0,1,2, \ldots, \\
M_{p} & =J_{p}, J_{p}-2, J_{p}-4, \ldots,-J_{p} .
\end{aligned}
$$

Analogously, the numbers $J_{l}$ and $M_{l}$ are invariant under the action of the $U(l)$ subgroup of the $U(p)$ group $(l=1,2, \ldots p-1)$, and characterize the representation of the $U(l)$ subgroup.

The maximal set of commuting operators is given by the chain of operators

$$
\Delta\left(X^{p}\right), \widehat{M}_{p}, \Delta\left(X^{p-1}\right), \hat{M}_{p-1}, \ldots, \Delta\left(X^{2}\right), \hat{M}_{2}, \widehat{M}_{1} .
$$

and corresponds, in fact, to the Gel'fand-Tsetlins parametrization [9] for the degenerate series of representations.

${ }^{6}$ Strictly speaking, the region $0 \leqq \varphi^{l} \leqq 2 \pi, l=1,2, \ldots p$ is expressed in terms of $\phi^{l}$ in the form of a set of involved inequalities which define only a part of the region given by (A.12). Nevertheless, due to symmetry properties of the harmonic functions in the whole region (A.12) the normalization integrals can be taken over the whole region (A.12) if only the normalization constant is appropriately changed. In the constant $N_{p}$ (see (2.22) and (A.17)) this change has already been taken into account. 
Expressing now our basic functions (A.8) in terms of the new variables, we obtain

$\mathbf{Y}_{M_{1}, \ldots, M_{p}}^{J_{2}, \ldots, J_{p}}(\Omega)=\frac{1}{\sqrt{N_{p}}} \prod_{l=1}^{p} \exp \left(i \mu_{l} \phi^{l}\right) \cdot \prod_{k=2}^{p} \sin ^{2-k} \vartheta^{k} d_{\alpha_{k}, \beta_{k}}^{\frac{1}{2}\left(J_{k}+k-2\right)}\left(2 \vartheta^{k}\right)$

where

and

$$
\begin{aligned}
\mu_{l} & =M_{l}-\frac{l}{p} M_{p} \quad l=1,2, \ldots, p-1 \\
\mu_{p} & =\frac{1}{p} M_{p} \\
\alpha_{k} & =\frac{1}{2}\left(M_{l}-M_{k-1}+J_{k-1}+k-2\right) \quad k=2, \ldots, p \\
\beta_{k} & =\frac{1}{2}\left(M_{k}-M_{k-1}-J_{k-1}-k+2\right) \quad J_{1} \equiv M_{1} \\
\Omega & =\left\{\phi^{1}, \ldots, \phi^{p}, \vartheta^{2}, \ldots, \vartheta^{p}\right\}
\end{aligned}
$$

$$
\begin{gathered}
\left|M_{2}-M_{1}\right|+\left|M_{1}\right|=J_{2}-2 n_{2} \quad n_{2}=0,1,2, \ldots, \frac{J_{2}-\left|M_{2}\right|}{2} \\
k=2,3, \ldots, p \\
\left|M_{k}-M_{k-1}\right|+J_{k-1}=J_{k}-2 n_{k} \quad n_{k}=0,1,2, \ldots \frac{J_{k}-\left|M_{k}\right|}{2} \\
N_{p}=(2 \pi)^{p} p \prod_{k=2}^{p}\left(J_{k}+k-1\right)^{-1} .
\end{gathered}
$$

This form of the basic harmonic functions has been used in Sections 2 and 3 .

Let us now discuss in more detail the series of representations of the $U(p)$ group which has been obtained. Each representation is determined
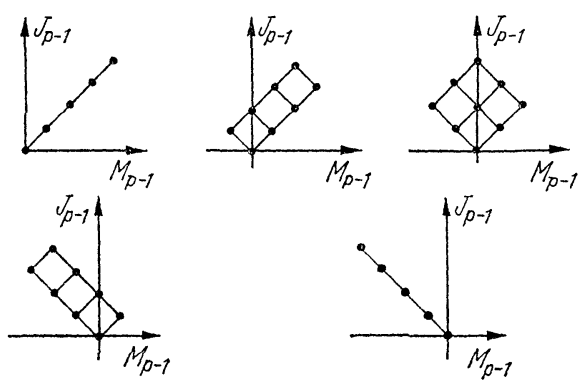

Fig. 7. Examples of nets representing admissible values of $J_{p-1}$ and $M_{p-1}$ in the representations $D_{M}^{L}\left(X^{p}\right), p>2$

by two integers $J_{p}$ and $M_{p}$ satisfying the conditions (A.14) and will be denoted by $D_{M_{p}}^{J_{p}}\left(X^{p}\right)$ or, briefly, $D_{M_{p}}^{J_{p}}$. It is convenient to represent each representation $D_{M_{p}}^{J_{p}}$ by a diagram in which each possible value of $\left(J_{p-1}, M_{p-1}\right)$ is denoted by a point. The diagrams are obtained by making 
use of the conditions

$$
\begin{gathered}
M_{p-1}=J_{p-1}-2 n \quad n=0,1, \ldots, J_{p-1} \\
\left|M_{p}-M_{p-1}\right|+J_{p-1}=J_{p}-2 n^{\prime} \\
n^{\prime}=0,1,2, \ldots, \frac{J_{p}-\left|M_{p}\right|}{2} \quad(p>2)
\end{gathered}
$$

which follow from (A.17). Some typical examples are shown in Fig. 7. If $p$ equals 2 , we obtain, instead of (A.18), the condition

$$
\left|M_{2}-M_{1}\right|+\left|M_{1}\right|=J_{2}-2 n \quad n=0,1, \ldots \frac{J_{2}-\left|M_{2}\right|}{2}
$$

\section{Irreducibility}

The Lie algebra $R_{p}$ of the unitary group $U(p)$ is a compact subalgebra of the $R_{p, q}$ algebra defined in Section 4 and its basis can be created from the operators $L_{i}, L_{i j}^{+}$and $L_{i j}^{-}(i, j=1,2, \ldots p, i<j)$, which satisfy the commutation relations given in the first row of (4.1).

The action of these operators on the set of harmonic functions with the basis (A.8) can be obtained if we represent the Lie algebra $R_{p}$ by the Lie algebra $\widetilde{R}_{p}$ of the first-order operators of differentiation with respect to $\varphi^{1}, \ldots, \varphi^{p}, \vartheta^{2}, \ldots, \vartheta^{p}$.

The irreducibility will be proved if we show that the Lie algebra $\widetilde{R}_{p}$ is irreducible on the Hilbert space $\mathscr{H}_{M_{p}}^{J_{p}}\left(X^{p}\right)$ of the harmonic functions (A.8).

We shall perform the proof by induction. The irreducibility is well known for the representations of $U(1)$ and $U(2)$ (see, e.g., [10]). Assuming now the irreducibility of $\widetilde{R}_{p-1}$ on the corresponding subspace $\mathscr{H}_{M_{p} M_{p-1}}^{J_{p} J_{p-1}}$ of the space $\mathscr{H}_{M_{p}}^{J_{p}}$ we shall show that the representation $\widetilde{R}_{p}$ of $R_{p}$ is irreducible on the space $\mathscr{H}_{M_{p}}^{\boldsymbol{J}_{p}}$.

The case $p>2$. The Hilbert space $\mathscr{H}_{M_{p}}^{J_{p}}$ has the following structure:

$$
\mathscr{H}_{M_{p}}^{J_{p}}=\sum_{J_{p-1}=0}^{J_{p}} \sum_{M_{p-1}} \oplus \mathscr{H}_{M_{p}, M_{p-1}}^{J_{p}, J_{p-1}}
$$

where the summation over $M_{p-1}$ is restricted by

$$
\begin{gathered}
M_{p-1}=J_{p-1}-2 n \quad n=0,1, \ldots J_{p-1} \\
\left|M_{p}-M_{p-1}\right|+J_{p-1}=J_{p}-2 n^{\prime} \quad n^{\prime}=0,1, \ldots \frac{J_{p}-\left|M_{p}\right|}{2}
\end{gathered}
$$

which can be represented with the help of diagrams; each diagram represents one space $\mathscr{H}_{M_{p}}^{J_{p}}$ and each point of a given diagram denotes one of its subspaces $\mathscr{H}_{M_{p}, M_{p-1}}^{J_{p}}$ (see Fig. 7).

To prove that there is no subspace of $\mathscr{H}_{M_{p}}^{J_{p}}$ invariant under the action of $\widetilde{R}_{p}$ it is sufficient to find some operator $L \in \widetilde{R}_{p}$ and one element $\mathbf{Y}_{M_{1}, \ldots, M_{p}}^{J_{2}, \ldots, J_{p}}(\Omega)$ of $\mathscr{H}_{M_{p}}^{J_{p}}$ such that $L \mathbf{Y}_{M_{1}, \ldots, M_{p}}^{J_{2}, \ldots, J_{p}}$ has nonvanishing components in all neighbouring subspaces, i.e., in all neighbouring points $J_{p-1} \pm 1$, $M_{p-1} \pm 1$ of the diagram under consideration (see [8]). 
To show this, let us find the action of the operators $L^{ \pm} \in \widetilde{R}_{p}$ where

$$
\begin{aligned}
L^{ \pm}= & L_{p, p-1}^{+} \pm i L_{p, p-1}^{-}=e^{\mp i\left(\varphi^{p-1}-\varphi^{p}\right)}\left[\operatorname { c o s } \vartheta ^ { p - 1 } \left(-\frac{\partial}{\partial \vartheta^{p}} \pm\right.\right. \\
& \left.\left. \pm i \operatorname{tg} \vartheta^{p} \frac{\partial}{\partial \varphi^{p}}\right)+\operatorname{ctg} \vartheta^{p}\left(\sin \vartheta^{p-1} \frac{\partial}{\partial \vartheta^{p-1}} \pm \frac{i}{\cos \vartheta^{p-1}} \frac{\partial}{\partial \varphi^{p-1}}\right)\right]
\end{aligned}
$$

on the basic vector

$$
\mathbf{Y}_{M_{p-2}, M_{p-1}, M_{p}}^{J_{p_{-2},}, J_{p_{-1},}, J_{p}} \equiv \mathbf{Y}_{0, \ldots, \ldots, 0, M_{p-2}, M_{p-1}, M_{p}}^{0, \ldots, J_{p-2}, J_{p_{-1}}, J_{p}}(\Omega)
$$

where for simplicity of calculation we choose $M_{p-2}=M_{p-1}$ and consequently, $J_{p-2}=M_{p-2}$. We obtain

$L^{ \pm} \mathbf{Y}_{M_{p-2}, M_{p-1}, M_{p}}^{J_{p-2}, J_{p-1}, J_{p}}=a^{+} b_{ \pm}^{+} \mathbf{Y}_{M_{p-2}, M_{p-1} \pm 1, M_{p}}^{J_{p-2}, J_{p-1}+1, J_{p}} \mp a^{-} b_{ \pm}^{-} \mathbf{Y}_{M_{p-2}, M_{p-1} \pm 1, M_{p}}^{J_{p-2}, J_{p-1}-1, J_{p}}$,

where $a^{ \pm}$and $b_{ \pm}^{ \pm}$have the following form:

$$
\begin{gathered}
a^{ \pm}= \pm \frac{\sqrt{\left(J^{\prime}-J^{\prime \prime} \pm 1\right)\left(J^{\prime}+J^{\prime \prime} \pm 1\right)}}{2 \sqrt{J^{\prime}\left(J^{\prime} \pm 1\right)}} \\
b_{+}^{ \pm} \equiv b_{+}^{ \pm}\left(m_{p}\right)=\sqrt{\left(J \pm J^{\prime}+1+m_{p}\right)\left(J \mp J^{\prime}-1-m_{p}\right)} \\
b_{ \pm}^{ \pm}\left(m_{p}\right)=b_{+}^{ \pm}\left(-m_{p}\right) \\
J=J_{p}+p-1, \quad J^{\prime}=J_{p-1}+p-2, \quad J^{\prime \prime}=J_{p-2}+p-3, \\
m_{p}=M_{p}-M_{p-1} .
\end{gathered}
$$

By successive application of the operators $L_{p, p-1}^{+} \pm i L_{p, p-1}^{-}$every point of the given diagram can be reached starting from an arbitrary point. Only two exceptional cases need a closer discussion, namely $M_{p-1}=J_{p-1}$ and $M_{p-1}=-J_{p-1}$. In the former case the simultaneous lowering of $M_{p-1}$ and $J_{p-1}$, which is allowed by the conditions (A.17), is impossible due to the fact that $J_{p-1}=J_{p-2}$ and that $a^{-}=0$. In this case we choose instead of (A.22), another basic vector $\mathbf{Y}^{\prime} \equiv \mathbf{Y}_{M_{p-1}-1, M_{p-1}, M_{p}}^{J_{p-1}-1, M_{p-1}, J_{p}}$ which differs from (A.22) only in the fact that the numbers $J_{p-2}=M_{p-2}$ are smaller by one in $\mathbf{Y}^{\prime}$. Acting by $L$ on $\mathbf{Y}^{\prime}$, we can perform the transition $M_{p-1}, J_{p-1} \rightarrow M_{p-1}-1, J_{p-1}-1$. The nonvanishing of this transition follows from the fact that

$$
\left(\mathbf{Y}_{M_{p-1}-1, M_{p-1}-1, M_{p}}^{J_{p-1}-1, J_{p-1}-1, J_{p}}, L-\mathbf{Y}_{M_{p-1}-1, M_{p-1}, M_{p}}^{J_{p-1}-1, J_{p-1}, J_{p}}\right)
$$

is equal to the complex conjugate of

$$
\left(\mathbf{Y}_{M_{p-1}-1, M_{p-1}, M_{p}}^{J_{p-1}-1, J_{p-1}, J_{p}}, L^{+} \mathbf{Y}_{M_{p-1}-1, M_{p-1}-1, M_{p}}^{J_{p-1}-1, J_{p-1}-1, J_{p}}\right)
$$

which is non-vanishing.

The case $M_{p-1}=-J_{p-1}$ is solved by the same method.

\section{Unitarity}

The unitarity of the representations $D_{M_{p}}^{J_{p}}\left(X^{p}\right)$ follows from the left invariance of the measure $d \mu\left(X^{p}\right)$ on the manifold $X^{p}$ analogously as in the case of non-compact groups $U(p, q)$ (see Section $4, \mathrm{II}$ ). 


\section{References}

[1] Helgason, S.: Differential geometry and symmetric spaces. New York: Academic Press 1962.

[2] Rosenfeld, B. A.: Dokl. Akad. Nauk SSSR 110, 23 (1956).

[3] Fedyenko, A. S.: Dokl. Akad. Nauk SSSR 108, 1026 (1956).

[4] Gel'fand, I. M.: Am. Math. Soc. Transl., Ser. 2, 37, 31-34 (1964).

[5] Rosenfeld, B. A.: Neevklidovy geometrii, Moscow 1955.

[6] Gel'fand, I. M., and M. I. Graev: Trudy Moscov. Matem. Obshchestva 11, 243 (1962).

[7] RaczKa, R.: Degenerate representations of compact and noncompact unitary groups, ICTP preprint, IC/65/80, Trieste.

[8] - N. Limić, and J. Niederte: Discrete degenrate representations of noncompact rotation groups, ICTP preprint IC/66/2, Trieste, to be published in J. Math. Phys.

[9] Gel'fand, I. M., and M. L. Tsetlin: Dokl. Akad. Nauk SSSR 71, 825, 1017 (1950).

[10] BÉG, M. A. B., and H. RuegG: J. Math. Phys. 6, 677 (1965). 